\title{
Ceftazidime treatment in cystic fibrosis: resistant organisms in sputum and faeces
}

\author{
A M Dalzell, D Sunderland, C A Hart, D P Heaf
}

\begin{abstract}
Ceftazidime was used as the only intravenous agent for treating lower respiratory tract infections in patients with cystic fibrosis. The risks of inducing $\beta$ lactamases and conferring antibiotic resistance are high when monotherapy is used; so the emergence of resistant bacteria was studied prospectively in the sputum of 120 patients. The mean age of patients was 9.0 (range $0.3-25$ ) years and there were equal numbers of male and female patients. Pseudomonas aeruginosa was the only ceftazidime resistant bacterium to be isolated from the respiratory tract, and was identified only in chronically colonised patients. Ceftazidime resistance occurred in $103(14 \%)$ of $750 P$ aeruginosa isolates, and in 16 of 36 chronically colonised patients. Ceftazidime resistant organisms were isolated from the faeces of 17 of 64 patients investigated. Eighty two per cent of the resistant faecal organisms were single isolates: the same resistant organism in faeces was isolated from successive samples in only two patients. In no case was the ceftazidime resistant enteric isolate the same as that from sputum. Patients chronically colonised by $P$ aeruginosa did not harbour ceftazidime resistant enteric organisms any more than non-colonised patients. The use of ceftazidime as a single intravenous agent in treating chest exacerbations in cystic fibrosis does not induce a reservoir of ceftazidime resistant bacteria.
\end{abstract}

Pseudomonas aeruginosa is frequently isolated from the oropharynx of patients with cystic fibrosis, ${ }^{1}$ and when it persists it is associated with an increase in morbidity. ${ }^{2}$ There is a strong association between bacteria found in the upper respiratory tract and lower airway flora, ${ }^{3}$ and in patients chronically colonised with $P$ aeruginosa it can rarely be eradicated despite treatment with broad spectrum antimicrobial drugs.

$P$ aeruginosa has several virulence factors that enhance its survival in cystic fibrosis. Selective intramural proteins ${ }^{4}$ are thought to inhibit antibiotic penetration, and alginate, produced by mucoid strains, probably protects against the host immune response. Pseudomonas also possesses inducible $\beta$ lactamases. These are chromosomally encoded enzymes that confer resistance against the $\beta$ lactam group of antibiotics and are produced in large amounts in response to certain $\beta$ lactam antibiotics. ${ }^{6}$

Conventional treatment of acute lower respiratory infections in patients colonised by $P$ aeruginosa is generally by a combination of antibiotics. The potential danger of using a single antipseudomonal antibiotic is that resistant strains might be selected. If this occurred, strains could rapidly develop resistance to widely varying $\beta$ lactam antibiotics, making treatment difficult and relapse more likely. ${ }^{7}$ Of the broad spectrum cephalosporins, ceftazidime has been shown to be as clinically effective as antibiotic combinations, and its use as a single agent has been advocated. ${ }^{8}$

If resistant strains of $P$ aeruginosa emerge in chronically colonised patients, the chest and gastrointestinal tract are the major potential reservoirs for such organisms. To determine whether ceftazidime monotherapy promoted the emergence of ceftazidime resistant organisms, we have conducted a prospective survey to see whether such bacteria are emerging in sputum and stool.

\section{Methods}

The study was conducted over two years and included all 120 patients attending Alder Hey cystic fibrosis clinic. The mean age of the patients was 9.0 (range $0.3-25$ ) years, with equal numbers of male and female patients. Thirty seven patients were less than 5 years, 37 were $5-9,23$ were $10-14$, and 23 were 15 or over. Clinical features supported by two positive results of sweat tests had confirmed the diagnosis of cystic fibrosis in each patient. Most patients $(85 \%)$ attended the outpatient clinic at intervals of four to six weeks.

At each hospital visit patients were assessed clinically and bacteriological culture of a sample of sputum or a throat or cough swab was undertaken. In addition, 64 patients submitted stool samples for culture.

\section{ANTIBIOTIC POLICY}

All patients received continuous flucloxacillin as prophylaxis against colonisation by Staphylococcus aureus. Acute respiratory infections were treated initially with amoxycillin, erythromycin, or trimethoprim, and if clinical improvement was not seen within seven days a clinical reassessment was made. If $P$ aeruginosa was isolated, the patient was treated with oral ciprofloxacin, nebulised aminoglycoside, or intravenous antibiotic. Ceftazidime was the 
only intravenous agent used $(150 \mathrm{mg} / \mathrm{kg}$ a day in three divided doses); it was given for 14 days either as a bolus or as a short period infusion. Inpatient treatment was within an open ward setting, and patients were isolated only when multiresistant $P$ aeruginosa or $P$ maltophila was identified. Once antipseudomonal treatment had been initiated, all other antimicrobial agents except flucloxacillin were discontinued.

\section{BACTERIOLOGICAL MONITORING}

Respiratory tract secretion samples (sputum after physiotherapy, cough swabs, or throat swabs) were taken at each clinic visit or while the patient was in hospital. Samples were cultured on blood agar, chocolate agar, and MacConkey agar. Colonies were picked and bacteria identified and tested for their susceptibility to a panel of antimicrobials (including ceftazidime) by a controlled disc diffusion method. If ceftazidime resistant bacteria were isolated, further samples were obtained to determine whether carriage or infection persisted.

Samples of faeces were obtained from patients before, during, and after ceftazidime treatment. Samples were applied to blood agar plates incorporating ceftazidime $(10 \mathrm{mg} / \mathrm{l})$. All bacteria growing on these plates were identified according to standard criteria ${ }^{9}$ and stored for determination of minimum inhibitory concentration (MIC). This was determined by an agar incorporation method with a bacterial inoculum of $10^{6}$ colony forming units and a range of concentrations $(1-64 \mathrm{mg} / \mathrm{l})$ of cefotaxime, ceftazidime, tobramycin, ticarcillin, and ciprofloxacin. If ceftazidime resistant isolates were made, stool samples were obtained more frequently to determine duration of carriage.

STATISTICAL ANALYSIS

Differences between patients were analysed by the Mann-Whitney $U$ test and the $\chi^{2}$ test.

\section{Results}

Oropharyngeal and lower airway bacterial flora were determined in 120 patients for the presence of ceftazidime resistant bacteria, and faecal flora in 64 . Sixty five patients $(54 \%$ of the clinic population) received 185 courses of ceftazidime during the study. One hundred and thirty three courses $(72 \%)$ were given to patients with chronic colonisation by $P$ aeruginosa (more than six months).

\section{RESPIRATORY FLORA}

Throat and cough swabs and sputum samples were cultured on 2480 occasions. The most frequent isolates were $P$ aeruginosa $(750 ; 30 \%)$, Haemophilus influenzae (370; 15\%), Staphylococcus aureus $(298 ; 12 \%), H$ influenzae plus $S$ aureus $(180 ; 7.5 \%)$, others $(228 ; 9.5 \%)$. No potentially pathogenic isolate was obtained in $654(26 \%)$ samples taken.

$P$ aeruginosa was isolated from the respiratory tract in 66 patients $(55 \%)$, of whom 36 $(30 \%)$ were chronically colonised (for more than six months). The mean age at which chronic $P$ aeruginosa colonisation was recognised was 10 (range 1-23) years.

The figure shows the results of 713 sputum samples (mean 20 per patient) cultured from the 36 patients with chronic $P$ aeruginosa colonisation. Each isolate was categorised as being resistant to aminoglycosides, carboxy and ureido penicillins, ciprofloxacin, or ceftazidime.

One hundred and three $P$ aeruginos $a$ isolates $(14 \%)$ were resistant to ceftazidime. They were isolated from the sputum of 16 chronically colonised patients, with a frequency of isolation of less than one in three samples collected. Forty five isolates were sensitive to all antibiotics but ceftazidime. All 58 multiresistant isolates $(6.3 \%)$ were from patients whose sputum yielded ceftazidime resistant bacteria. Two patients carried Pseudomonas

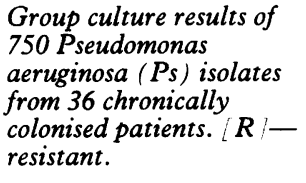

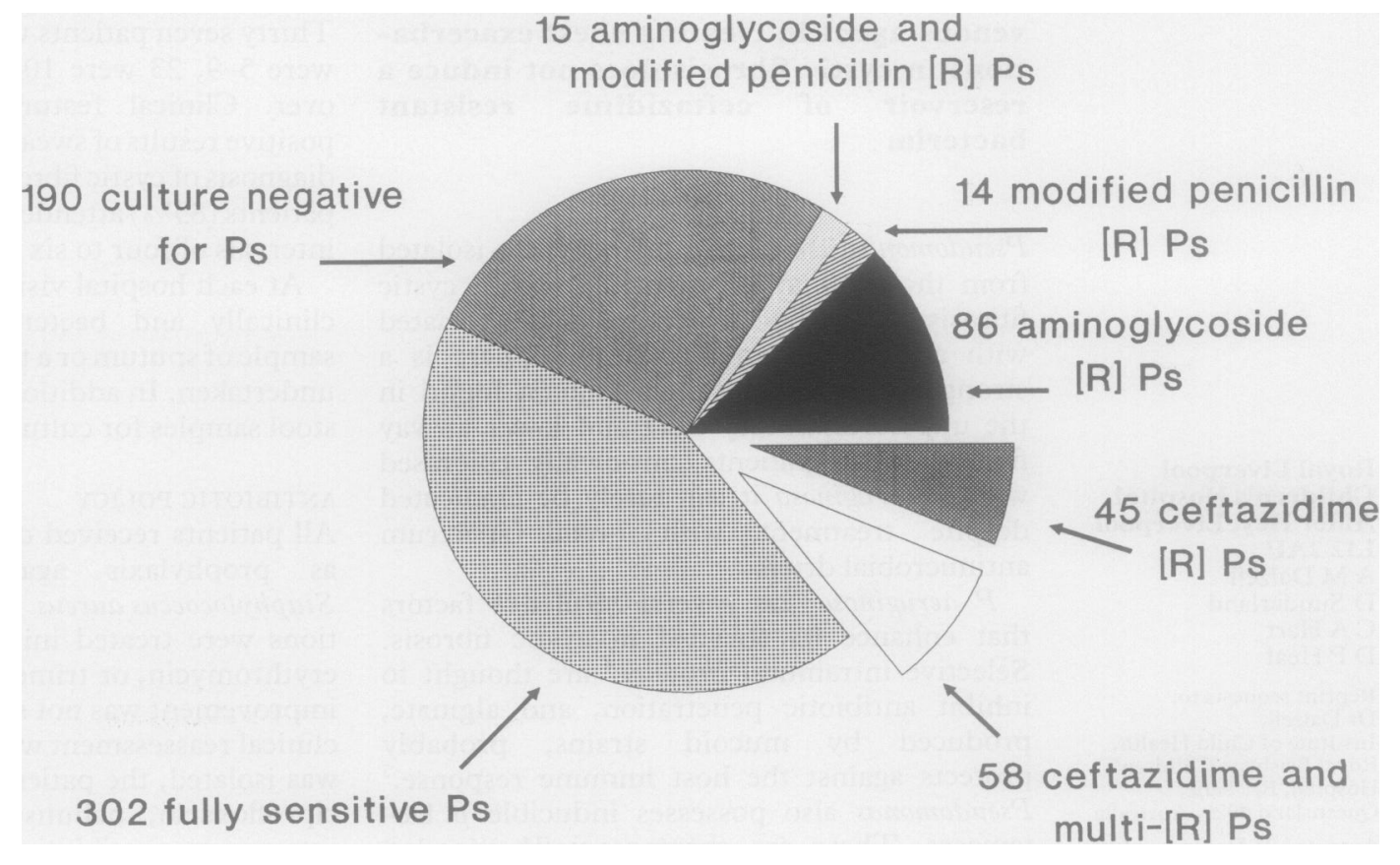


Comparison between patients chronically colonised with Pseudomonas aeruginosa who carry ceftazidime resistant bacteria in sputum and those who do not (means with ranges)

\begin{tabular}{|c|c|c|c|}
\hline & \multicolumn{2}{|l|}{ No of patients } & \multirow[b]{2}{*}{$p$} \\
\hline & $\begin{array}{l}\text { Ceftazidime } \\
\text { resistant } \\
P \text { aeruginosa } \\
(n=16)\end{array}$ & $\begin{array}{l}\text { Ceftazidime } \\
\text { sensitive } \\
P \text { aeruginosa } \\
(n=20)\end{array}$ & \\
\hline $\begin{array}{l}\text { Current age (years) } \\
\text { Current Shwachman score } \\
P \text { aeruginosa colonisation (years) } \\
\text { Ceftazidime usage (courses in } 2 \text { years) }\end{array}$ & $\begin{array}{l}15 \cdot 8(13-25) \\
53(35-80) \\
4 \cdot 8(0 \cdot 5-10) \\
5 \cdot 3(0-15)\end{array}$ & $\begin{array}{l}12 \cdot 4(1-24) \\
70(50-95) \\
2 \cdot 7(0 \cdot 5-15) \\
2 \cdot 4(0-6)\end{array}$ & $\begin{aligned} & 0.13 \\
< & 0.001 \\
< & 0.05 \\
< & 0.02\end{aligned}$ \\
\hline
\end{tabular}

maltophilia, which was not multiresistant, in addition to resistant $P$ aeruginosa.

At the mid point of the study period the 16 patients with chronic colonisation by ceftazidime resistant $P$ aeruginosa had lower Shwachman scores, had been colonised longer, and had had more courses of ceftazidime (table).

\section{FAECAL FLORA}

Of 127 faecal samples, 29 ceftazidime resistant bacteria were obtained from 17 of the 645 patients providing stool samples (127 samples in all). Of these 17 patients, three had never taken ceftazidime. Two patients carried two different ceftazidime resistant bacteria on separate occasions. Most isolations were made on one occasion only ( $82 \%)$; five patients, however, carried the same organism on more than one occasion, the longest period being 11 months before carriage ceased. Only five patients with chronic colonisation of the chest with $P$ aeruginosa carried a ceftazidime resistant bacterium in their intestine and in nò case was the same ceftazidime resistant bacterium isolated from faeces and the respiratory tract at the same time. There was no relation between the presence of ceftazidime resistant $P$ aeruginosa in sputum and in faeces $(\mathrm{p}=0 \cdot 7)$. The MICs of 22 of 29 ceftazidime resistant bacteria (Escherichia coli 9, Enterobacter spp 5, Citrobacter freundii 4 , and one each of $P$ aeruginosa, $P$ maltophila, Acinetobacter spp, and Hafnia alvei) isolated from faeces were all, except for one isolate, above $64 \mathrm{mg} / 1$ for ceftazidime and all had an MIC for ticarcillin above $64 \mathrm{mg} / \mathrm{l}$. All were sensitive to ciprofloxacin ( 20 with an MIC below $1 \mathrm{mg} / \mathrm{l}$ )

\section{Discussion}

In our patients with cystic fibrosis a persistent reservoir of ceftazidime resistant bacteria did not develop in either the respiratory or the gastrointestinal tract when ceftazidime was used as single agent treatment for $P$ aeruginosa infection.

The presence of ceftazidime resistant $P$ aeruginosa in the sputum of patients was related to greater use of ceftazidime, longer colonisation by $P$ aeruginosa, and a lower Schwachman score, but not to age. Patients treated more frequently with ceftazidime are more likely to carry ceftazidime in their sputum, though this occurred with a frequency of less than 1 in 3 in the samples we cultured. Ceftazidime resistant bacteria were found infrequently in faecal samples, and patients chronically colonised by $P$ aeruginosa were no more likely to carry ceftazidime resistant bacteria in their stool than were patients without respiratory tract colonisation.
These findings are encouraging, showing as they do a low prevalence of ceftazidime resistant organisms in patients who have had repeated exposure to the antibiotic over two years. The good clinical response and lack of important side effects ${ }^{10}$ make ceftazidime a suitable first line antibiotic in treating lower respiratory exacerbations in patients with cystic fibrosis. Ceftazidime combined with an aminoglycoside produces a more favourable quantitative reduction in the $P$ aeruginosa colony count than ceftazidime alone, but dual antibiotic treatment produces no detectable clinical advantage over ceftazidime treatment alone. ${ }^{8}$

This study does not address the question of the temporal relation between the occurrence of resistant isolates and treatment with ceftazidime. Schryvers et $a l^{11}$ reported $\beta$ lactamase production by $P$ aeruginosa in one patient with cystic fibrosis treated with ceftazidime, and noted that resistant strains of $P$ aeruginosa were isolated mainly during treatment. They suggested that bacteria susceptible to ceftazidime flourish before and after the treatment period and have a selective growth advantage. It is not surprising, therefore, that greater numbers of ceftazidime resistant isolates from sputum occurred in the chronically colonised patients who received the greatest number of ceftazidime courses during the study period.

The unique relation between $P$ aeruginosa and patients with cystic fibrosis makes interpretation of in vitro sensitivity studies particularly difficult and, although we have not presented data to show the clinical improvement with intravenous antibiotic use, we have found that a good clinical response is common even when ceftazidime resistant $P$ aeruginosa is isolated from sputum during treatment with ceftazidime. We change antibiotics only in the face of multiply resistant organisms and an absence of clinical improvement.

AMD held a Cystic Fibrosis Research Trust fellowship.

1 Friend PA. Pulmonary infection in cystic fibrosis. J Infect 1986;13:55-72.

2 Hoiby N. Microbiology of lung infections in cystic fibrosis patients. Acta Paediatr Scand 1982;301(Suppl):33-54.

3 Thomassen MJ, Klinger JD, Badger SJ, van Heeckeren DW, Stern RC. Cultures of thoractomy specimens confirm usefulness of sputum cultures in cystic fibrosis. J Pediatr usefulness of sputur

4 Nicas TI, Hancock REW. Pseudomonas aeruginosa outer membrane permeability: isolation of a porin protein $F$ deficient mutant. J Bacteriol 1983;153:281-5.

5 Martin DR. Mucoid variation in Pseudomonas aeruginosa induced by the action of phage. J Med Microbiol 1973;6:111-8.

6 Sanders CC. Medical perspective. Novel resistance selected by the new expanded-spectrum cephalosporins: a concern. J Infect Dis 1983;147:585-9.

7 Sanders CC, Sanders WE Jr. Emergence of resistance during therapy with the newer $\beta$-lactam antibiotics: role of inducible $\beta$-Lactamases and implications for the future. Rev Infect Dis 1983;5:639-48.

8 Padoan R, Cambisano W, Costantini D, et al. Ceftazidime monotherapy vs. combined therapy in Pseudomonas pulmonary infections in cystic fibrosis. Pediatr Infect Dis $J$ 1987;6:648-53.

9 Cowan ST, Steel KJ. Manual for the identification of medical bacteria. 2nd ed. Cambridge: University Press, 1974: $1-238$.

10 Dodge J, Zamiri I, Goodchild M, Ingram P. Experience with ceftazidime in cystic fibrosis. $J$ Antimicrob Chemother with ceftazidim

11 Schryvers AB, Ogunariwo J, Chamberland S, Godfrey AJ, Rabin HR, Bryan LE. Mechanism of Pseudomonas aeruginosa persistence during treatment with broad-spectrum cephalosporins of lung infections in patients with cystic fibrosis. Antimicrob Agents Chemother 1987:31:1438-9. 